\title{
Efectos del Cumplimiento del Ramadán Durante el Embarazo sobre el Peso al Nacer con datos de África
}

\author{
María Natalia Cantet
}

\author{
Tesis de Maestría \\ Maestría en Economía \\ Universidad Nacional de La Plata
}

Director de Tesis: Prof. María Laura Alzua

29 de Agosto de 2014

Códigos JEL: I15, J13, O15, I12. 


\section{Agradecimientos}

Esta tesis fue posible gracias a los generosos aportes de la Facultad de Ciencias Económicas de la Universidad de la Plata, provincia de Buenos Aires, y en particular al Centro de Estudios Distributivos Laborales y Sociales (CEDLAS). Además extiendo mi agradecimiento a Carolina Lopez por la ayuda brindada, pero, sobre todo, a la Profesora Dra. María Laura Alzua, que generosamente donó su tiempo y su conocimiento para que el presente trabajo pueda ser realizado en tiempo y forma.

Y a mi familia por su inagotable paciencia y amor 


\section{Índice}

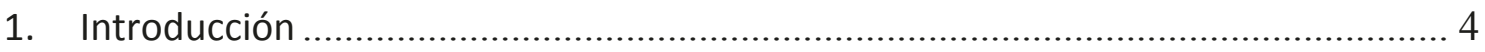

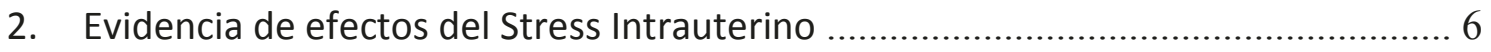

2.1 Explicación biológica del impacto del ayuno ................................................. 8

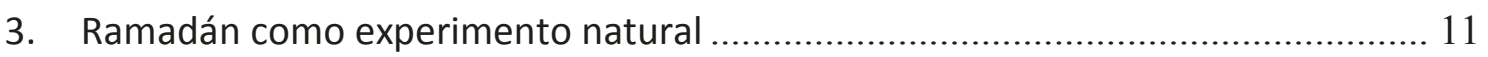

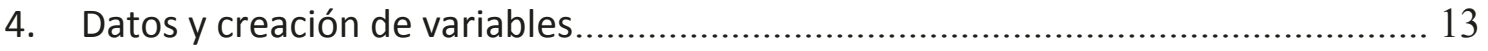

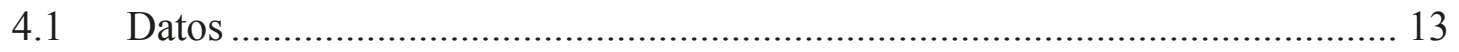

4.2 Medidas de Exposición al Ramadán ................................................................ 16

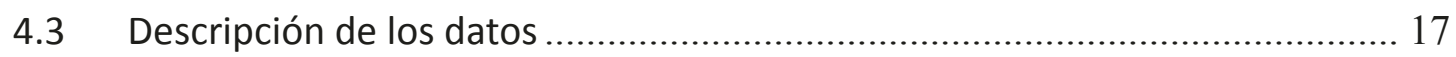

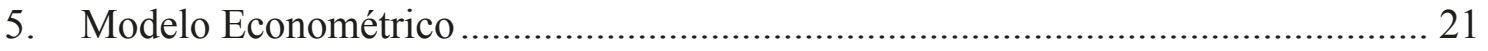

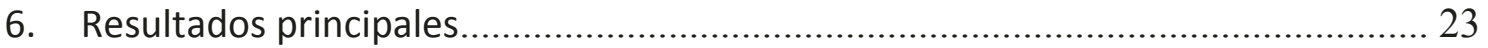

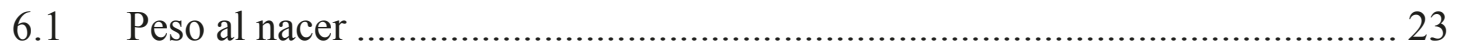

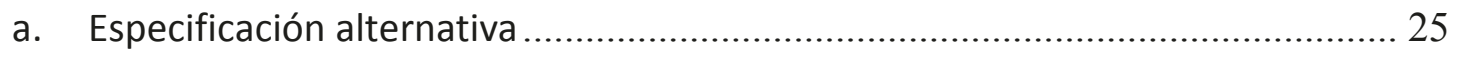

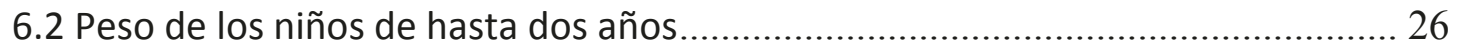

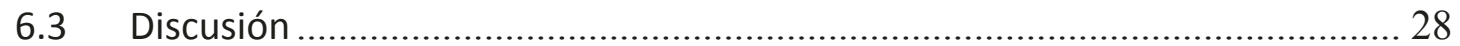

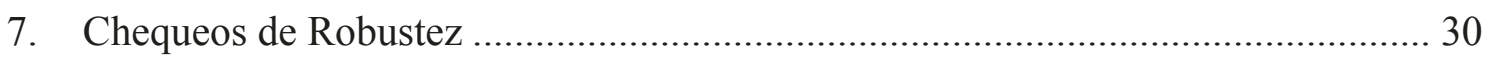

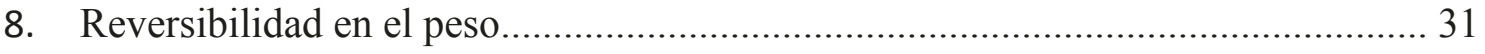

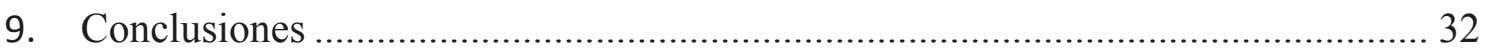






\section{Introducción}

El peso al nacer es una de las medidas más utilizada como proxy de la salud y bienestar infantil en la literatura económica. Aquellos niños con bajo peso al nacer (LBW), convencionalmente definido como menos de 2500 gramos, suelen experimentar efectos severos sobre la salud, la mortalidad, la morbilidad y la discapacidad. Por otro lado, el bajo peso al nacer también se traduce en costos sustanciales en el sector de la salud e impone una carga significativa sobre la sociedad en su conjunto. El desarrollo corporal de las madres al igual que su estilo de vida determinarán el peso del bebé al momento de nacer.

Resulta posible listar los factores determinantes del peso al nacer como los efectos genéticos maternos o predisposición (sin incluir factores genéticos por parte del progenitor o padre), el deficiente cuidado prenatal, factores ambientales o inclusive el parto prematuro. Sin embargo, el hecho de que muchos factores no sean observables limita la posibilidad de aislar el efecto propio de cada uno. En este sentido, el impacto de eventos que provocan "estrés" en el embrión durante el período de gestación no es la excepción.

Este estudio se centra en el desarrollo de la vida fetal y explora cómo la desnutrición en el útero afecta al peso al nacer y durante los primeros años de vida mediante el uso de las fechas exactas de nacimiento de los niños menores de 5 años y la explotación de la naturaleza calendárica de la incidencia de las déficits nutricionales derivadas de la exposición con el mes de ayuno musulmán que ocurre durante el Ramadán. La estrategia de identificación econométrica utiliza a las variaciones en el tiempo de exposición al Ramadán durante la gestación y la religión para identificar el efecto el peso.

La Organización Islámica Argentina define al Ramadán como un período en el que, entre la salida y la puesta del sol, todos los musulmanes que estén en condiciones físicas y sean adultos deben abstenerse de comer, beber, fumar y tener actividad sexual. De acuerdo con esta definición quedan excluidos los niños, los ancianos, los enfermos, quienes viajen, las mujeres embarazadas, y las madres que acaban de dar a luz. Sin embargo, en caso de formar parte de alguno de estos grupos (exceptuando los dos primeros) es necesario pedir una dispensa especial y recuperar los días cuando se retorne a la normalidad. Vale aclarar también que la duración del citado ayuno es de 29 
o 30 días, y la fecha depende del calendario lunar, no teniendo entonces un momento fijo en el año gregoriano.

Otro posible comportamiento con potencial peligro para el correcto desarrollo fetal relacionado con el Ramadán es la negación a ingerir medicamentos prescriptos, la deshidratación o vómito excesivo mientras se ayuna. Asimismo, el consumo de un gran número de dulces por la tarde pueden llevar a picos en el nivel de glucosa en sangre causando anormalidades en los niños. Este conjunto de comportamientos suelen darse en ocasiones en las que el desorden es general. En las comunidades musulmanas, aunque algunas mujeres musulmanas no dejen de alimentarse de forma apropiada en esta parte del embarazo, un entorno que sí procede a realizar el ayuno diurno durante el Ramadán puede provocar trastornos alimenticios y del sueño. Esto es probablemente la principal fuerza impulsora de esta deficiencia.

Este trabajo contribuye a la literatura de peso al nacer puesto que utiliza un Panel largo de los 37 países de África con información disponible sobre religión y peso al nacer en las Demographic Health Surveys. Esto conforma un panel no balanceado con más de 50 mil madres y 264 mil observaciones. Se llegó a la conclusión de que el efecto es mayor en países en los cuales el Ramadán es cumplido por un mayor porcentaje de personas.

El presente estudio tiene también implicaciones para la creación de políticas Por un lado, ayunar colectivamente crea un ambiente que genera desorden alimenticio persistente para las mujeres embarazadas. Una diferencia fundamental con la literatura de hambruna, es que estas condiciones pueden tener efectos negativos para la salud a largo plazo para un feto, como la pérdida de peso. Por lo tanto, desde el punto de vista de la formulación de políticas, se pone de relieve la importancia de proporcionar información al público sobre los riesgos específicos del trastorno de la dieta materna durante el embarazo.

Este estudio se organiza de la siguiente manera. En la siguiente sección se realiza un breve repaso de la literatura existente (tanto económica como médica). La sección 3 se refiere a los costos relacionados con niños con bajo peso al nacer en el continente africano y en la 4 se explica bajo qué condiciones es posible considerar al mes del Ramadán como experimento natural. Luego, en la sección 5 se realiza una presentación de los datos utilizados en este trabajo, mientras que en la siguiente se establece el modelo. En la sección 7 se describen a los resultados y en la 8 se evalúa si la hipótesis sobrevive a diferentes especificaciones. Por último, en la parte 9 se analiza la 
perdurabilidad del efecto de ayunar durante la gestación en el peso de niños de 5 años y en la 10 se concluye.

\section{Evidencia de efectos del Stress Intrauterino}

Numerosos autores han detectado una relación negativa entre diversas situaciones de estrés durante el período de gestación y variables relacionadas con la salud y los resultados socioeconómicos tanto en el corto como en el largo plazo. Por un lado, este estrés puede ser provocado por una situación relacionada con aspectos de la persona, como la religión o las costumbres de la madre, o por otro lado a causa de factores ajenos relacionados con el contexto.

Las situaciones traumáticas como una guerra o una crisis pueden tener consecuencias sobre el desarrollo intrauterino. Camacho (2008) evaluó el efecto del estrés psicológico prenatal causado por los ataques terroristas aleatorios de minas terrestres en la región de residencia de la familia sobre el peso al nacer y nacimiento pre-término. El estudio comprendió más de cuatro millones de nacimientos y abarcó1200 municipalidades colombianas entre 1998 y 2003, incluyendo múltiples partos por madre. El mismo determinó que un brote de violencia aleatorio durante el primer trimestre del embarazo tenía un impacto significativo en el peso al nacer de los niños, precisamente 8,7 gramos menos. Esta consecuencia persistió aun cuando se incluyeron los efectos fijos por madre, por lo que es posible inferir que no existen características maternas, observables o no, que expliquen el resultado.

En otro estudio, Cruces, Gluzman y López Calva (2011) se intenta establecer el efecto quela crisis más reciente de Argentina, en el año 2001, tuvo sobre el peso al nacer y los resultados escolares. Según los autores, los efectos directos como el trabajo y el ingreso, son los primeros en recuperarse por lo que los efectos relacionados con el capital humano se ven confundidos entre recesiones y recuperaciones. Es por esto que en la investigación, se toma la variación interprovincial e intertemporal del producto interno bruto (PIB) regional y varios otros resultados para el período 1993-2003 como estrategia de identificación de los cambios exógenos del momento. El resultado principal indica efectos sustanciales en el peso al nacer y la mortalidad, y un efecto 
contra cíclico (pero no significativo) en la matrícula escolar. Este efecto es asimétrico: el efecto negativo de una crisis es mayor que la recuperación en el período siguiente.

Por otro lado, ciertos comportamientos o aspectos de la vida cotidiana también pueden generar estrés en el desarrollo intrauterino y en consecuencia, afectar variables en el corto y el largo plazo. Algunos ejemplos de comportamientos peligrosos durante el período de gestación podrían ser fumar, desorden alimenticio, o ayunar por largos períodos de tiempo.

Uno de los factores de más riesgo durante el embarazo, y el principal en los Estados Unidos, para el peso al nacer y la mortalidad es el tabaquismo. Tomando una población de partos simples, Almond (2004) intenta aislar este efecto sobre diversas mediciones de salud en la niñez controlando por variables indicativas de su ambiente. El efecto aproximado es de 200 gramos y la probabilidad de mortalidad aumenta en aproximadamente un 0,08 .

Esta tesis, sin embargo, está centrada mayormente con la más reciente literatura que utiliza las características particulares del mes sagrado para identificar los efectos adversos de la malnutrición intrauterina en el peso al nacer, vulnerabilidad a enfermedades crónicas y discapacidades a una edad avanzada, el salario en la adultez y la educación (Almond 2004, van Ewik 2011, Almond et al. 2011, Majid 2012 y Karimi 2014). Estos estudios se basan en la teoría de los orígenes que toma a las variaciones en la variable peso al nacer como un indicio de modificaciones del desarrollo atribuidas a la mala nutrición intrauterina.

Almond y Mazunder (2011) fueron los primeros que analizaron el efecto del cumplimiento del Ramadán por parte de las mujeres embarazadas sobre el peso al nacer de sus hijos. Tomando a través de varias medidas de exposición al tratamiento durante el embarazo en la ciudad de Michigan (Estados Unidos), Uganda, e Irak (siendo el número de días de correspondencia y cantidad de horas de luz solar las más destacadas). Considerando a cada país por separado, observaron una mayor incidencia cuando la mujer realizó el Ramadán en el primer trimestre del embarazo. Además, evaluaron la posibilidad de que el ayuno afectara la composición de los niños nacidos en cuanto a la cantidad de hombres y mujeres. A tal fin, regresaron el ratio de la cantidad de Mujer versus la de hombres en las medidas de exposición al Ramadán. Sus resultados indican que el séptimo mes de gestación tiene un efecto negativo y significativo.

Existen investigaciones subsiguientes que analizan diversos efectos del cumplimiento del Ramadán durante el embarazo. Además de afectar el metabolismo del feto, el 
ambiente intrauterino, y la nutrición en particular, pueden conducir a futuros problemas de salud para adultos. Tomando como referencia a Indonesia, que es uno de los países en desarrollo con mayor cantidad de población musulmana (y un significativo $12 \%$ de individuos no-musulmanes), Ewik (2004) evaluó los efectos de la exposición al Ramadán por parte de la madre durante su embarazo y sobre la salud en la adultez. Su principal conclusión es que existe evidencia de una desmejora en la salud en general, un menor ratio de Mujer-Hombre y una mayor incidencia de enfermedades cardíacas coronarias, diabetes tipo II y anemia en la tercera edad y obesidad. Para el mismo país, Majid (2012) estimó los efectos del mismo comportamiento de largo plazo y en el mercado laboral. Llega a la conclusión de que los adultos expuestos entre los 15 y 65 años trabajaban menos horas y eran más proclives a trabajar por cuenta propia. Asimismo, los niños expuestos obtuvieron calificaciones más bajas y mayor posibilidad determinar realizando trabajo infantil.

Por último, y más ligado a cuestiones metodológicas, este trabajo también se encuentra directamente asociado la investigación de Karimi (2014) puesto que el autor estudió la estatura como indicador de desarrollo en un panel utilizando 27 cohortes de 35 países de África en cuya población musulmana es significativa. La principal conclusión es que, al margen del trimestre de ocurrencia, aquellos varones totalmente expuestos al mes de malnutrición (es decir, 30 días completos) sufren una reducción aproximada de entre 3,5 y 4,5 milímetros a entre los 3 y 4 años de edad. Sin embargo, no se encuentran efectos para las mujeres. Por otro lado, la exposición comienza a tener un efecto significativo cuando el Ramadán se superpone por más de 10 días con la gestación. Además, no se encontraron efectos provenientes de la latitud ni transmitidos por la genética.

\subsection{Explicación biológica del impacto del ayuno}

Desde un punto de vista médico, la explicación acerca de cómo el comportamiento asociada al Ramadán afecta al niño al nacer está estrechamente ligada a las teorías del ayuno. Bajo condiciones estándares, este comportamiento reduce el suplemento de glucosa que es una de las principales fuentes de energía. Luego, para compensar esta falta el cuerpo intenta acelerar el proceso de metabolismo (hipoglucemia). Dicho fenómeno, combinado con la falta de nutrientes, puede traerle graves daños al feto puesto que las mujeres embarazadas corren un riesgo mayor de entrar en un estado de 
hipoglucemia o de alto nivel de otras hormonas relacionadas. En el ambiente médico se denomina a este fenómeno como accelerated starvation (inanición acelerada) por la mayor velocidad con la que las futuras madres llegan a sufrir hipoglucemia.

Cabe señalar que accelerated starvation ocurre simplemente si una mujer embarazada no cena un día y se desayuna al día siguiente. E incluso este es más rápido cuando el ayuno es diurno que es precisamente el comportamiento esperado durante el Ramadán. En este ambiente hostil o de estrés, se pondrá en marcha un proceso de adaptación durante la gestación (fetal) que buscará minimizar los daños o posponerlos para períodos más tardíos de la vida, para adaptarse a los cambios en el metabolismo. Los teorizadores incluso estiman que el cuerpo "intentará" mantenerse vital hasta la etapa reproductiva, período después del cual comenzará a sufrir las consecuencias de esa adaptación.

Almond y Mazumder (2011) explicaron que existe una heterogeneidad en el impacto del ayuno según el período de la gestación. Tanto el mes como la variable de estudio (niveles educativos, ayuno, cuestiones sanitarias, etc.) impactan de forma my diferente a resultados hallados. En el caso de peso al nacer, efectos varían dependiendo del canal y de la muestra. Por ejemplo, el ayuno conduce a niveles bajos de glucosa en la sangre que a su vez, puede llevar al bajo peso al nacer en el tercer trimestre, o el final del segundo trimestre cuando se lo asocia con una gestación más corta.

\subsection{Relevancia Económica del Bajo Peso al Nacer (LBW)}

En las secciones previas se observó la relación negativa que existe entre el ayuno o el desorden alimenticio asociado al sagrado mes del Ramadán. Aquí se propone examinar el impacto para los hogares de tener bebes que sufren bajo peso al nacer o niños con algún tipo de deficiencia en el peso durante la edad temprana. Esto se vuelve particularmente relevante puesto se trata de países en desarrollo del continente africano donde tanto la infraestructura de las viviendas como la de bienes públicos, la infraestructura: carreteras, hospitales y servicios son deficientes. Por lo tanto, si bien podría tratarse de montos pequeños, es posible que tengan implicancias mayores a lo esperado en otras partes del mundo.

Dada la variedad y la extensión de las posibles consecuencias de la inanición acelerada resulta posible aproximarse al impacto monetario y sobre el bienestar. A tales fines, y 
partiendo de una perspectiva médico-familiar en Alemania, Hade et al. (2011) realizan un meta-estudio sobre las consecuencias económicas de los partos prematuros, haciendo particular foco en el bajo peso al nacer. Los autores dividen a los costos de corto plazo en cuatro categorías distintivas:

- Costos médicos directos, relacionados con, por ejemplo, posibles internaciones, visitas a especialistas, medicamentos y todo lo relacionado con los gastos en materia de salud.

- Costos no-médicos directos, ligados a los cuidados del niño que no son necesariamente médicos, como el transporte, ayuda extra en el hogar, cuidado de otros niños en la familia, entre otros.

- Costos indirectos, como las pérdidas de ingresos percibidas por los principales tutores por posibles cambios en sus status laboral o mayor nivel de ausentismo en el trabajo, e incluso el costo de oportunidad de la horas dedicadas al cuidado del niño.

- Costos intangibles que afectan directamente a la calidad de vida de los niños y de sus familiares directos en el mediano o largo plazo.

Desafortunadamente, no existen muchos datos acerca de los costos médicos directos del LBW para países de ingreso bajo (o medio) en los cuales el sistema de salud suele ser débil. Almond (2004) estudia como afectó el consumo de tabaco en el gasto del cuidado de la salud. En particular, cuantificó los costos médicos para los niños con muy bajo peso al nacer (menos de 1500 gramos) para los Estados Unidos en US\$100.000 (en dólares del año 2000). Incluso niños con LBW entre 2000 gr y 2500 gr conllevan costos de alrededor de US\$10.000 para el mismo país.

Por otra parte, es importante mencionar que existen incluso pocos estudios que tratan de cuantificar los costos asociados al LBW para África, En busca de aproximarse más a las condiciones del continente, Tongo et al. (2008) y Sicuri et al. (2011) realizaron estimaciones similares a las realizadas por Almond, pero para Nigeria y un área rural del Sur de Mozambique respectivamente.

En el trabajo de Sicuri et al. (2011) se realiza una aproximación a este monto para un hospital de una comunidad rural del Sur de Mozambique. Monitorearon a 120 niños con LBW de 3300 niños nacidos en la institución y presenta los gastos médicos directos e 
indirectos para los hogares. El tratamiento estándar de los niños es en promedio US $\$ 24,12$, en donde un aumento de 100 gramos implica un incremento del 53\% del total. Además, cada hospitalización agrega US\$8,50 en términos de gastos en salud y relacionados. Por contrapartida, en la provincia de Maputo el sistema de salud debió aportar US\$169.957,61 durante 2007 para el cuidado de la salud de los 120 niños nacidos con $\mathrm{LBW}^{1}$, situación que se da por cada 1000 niños nacidos.

En otro estudio similar, Tongo et al. señalan que los costos de hospitalizaciones tienen una media aproximada de US\$247,3 lo que representa un $22 \%$ del salario promedio.

Los efectos de largo plazo sobre la calidad de vida son variados en términos de las dimensiones que abarcan. En este sentido, la investigación realizada por Ewijk se concentra en los efectos de largo plazo de la confluencia entre el Ramadán y la gestación en la población musulmana en Indonesia. Observa que dicha población, tiene en general una salud más deficiente, efecto que se profundiza con la edad y esto puede, incluso, llegar a manifestarse únicamente en la adultez.

\section{Ramadán como experimento natural}

Al utilizar al sagrado mes del Ramadán, es claro que el estrés intrauterino es causado por la malnutrición $y$, por otro lado, la estimación de la fecha de nacimiento permite establecer el momento exacto (medido como trimestre o mes) en el que se produjo, y en el período medido en días de confluencia, la superposición entre la gestación y el mes de estudio. Por lo tanto, dada la información detallada acerca de la religión y las madres, puede aislarse con cierta exactitud el efecto buscado.

Cabe señalar, además, que Almond y Mazunder (2011), Majid (2012) y los estudios subsiguientes no encontraron evidencia que sustente la hipótesis de autoselección relacionada con la fecha de la concepción. Es decir, no hay evidencia que las madres eligieron el momento oportuno de modo de no hacer coincidir la gestación con el mes del Ramadán. Por lo tanto, para el presente trabajo se realizó el mismo supuesto de identificación: la elección del momento de la concepción (y por consiguiente, el

\footnotetext{
${ }^{1}$ Estos gastos fueron contabilizados por hospital sobre 3.332 nacimientos y constan de gastos de rutina (US\$50.953,63), las admisiones incremental a otros hospitales (US\$29.937,60) y provenientes de otros hospicios (US\$4.145,43), internaciones durante el primer año de vida (US\$11.612,16) y transporte a terceros (US\$4.689,48).
} 
embarazo), no está relacionada con la fecha del Ramadán y, en consecuencia, se elimina la posibilidad de autoselección por medio de la elección de la fecha de concepción.

Otra cuestión que debe examinarse está relacionada con la relación entre la exposición y el cumplimiento del ayuno pertinente. Este punto es de vital importancia para el estudio puesto que las conclusiones están directamente relacionadas. Como ya expuesto previamente, cabe recordar que si bien las mujeres embarazadas pueden no realizar el ayuno del mismo modo que los demás familiares, es necesario pedir una dispensa especial y dado que se debe realizar el ayuno al retornar a la normalidad la carencia de incentivos hace que no se solicite el mencionado permiso. Varios estudios reflejan que el ayuno en la cultura del Islam durante Ramadán es usual. En Singapur, Joosoph et a-l (2004) realizaron una encuesta a 202 mujeres elegibles, provenientes de diferentes estratos sociales, y llegaron a la conclusión de que el ayuno no es considerado como algo dañino y que muchas mujeres lo cumplen al menos un día. Para ellas, es preferible tomar el compromiso con sus familias para no tener que efectuarlo luego en soledad. En consonancia con esto, Ewijk (2011) observó que el cumplimiento es considerado como un hábito en Indonesia y que la mayoría actúa en concordancia, a pesar de reportarlo cómo difícil de realizar. Almond y Mazunder (2011) estimaron que entre el 70\% y el $90 \%$ de las mujeres musulmanas embarazadas cumplen con el rito alrededor del mundo. Debe notarse también que es más probable que el ayuno sea efectuado cuando coincide con los primeros meses de la gestación, ya sea por desconocimiento o por una mayor movilidad de la madre que lo facilita.

No obstante, Majid (2012) señaló que aun cuando la madre decida no adherir al ayuno, tal cual lo dicta la Religión Musulmana, es común que ellas experimenten cierto desorden y restricciones en la alimentación. Los supermercados y los restaurants suelen permanecer cerrados durante todo el mes. En sus hogares, las mujeres musulmanas establecen por lo general las comidas principales antes del amanecer y luego del atardecer lo cual conlleva privación de sueño. De este hecho se desprende que las mujeres musulmanas observan el Ramadán incluso cuando conscientemente deciden que no lo harán.

Finalmente, y si bien no es posible observar el grado del ayuno a partir de la base de datos disponible, el cumplimiento es fundamentalmente un rito del Islam y no es probable que sea un hábito extendido a otras religiones. Esto implica que los estimadores serán efectos intent to treat (ITT). Se compararán los pesos al nacer de niños cuyas gestaciones coincidieron, al menos en parte, con el mes del Ramadán. Es 
por esta razón que debe considerarse a los resultados ITT como una estimación conservadora del efecto, o como su límite inferior ${ }^{2}$.

\section{Datos y creación de variables}

\subsection{Datos}

Para el análisis empírico se utilizaron las Demographic and Health Survey Data (DHS) de acuerdo con su disponibilidad, entre 1984 y 2012 para el continente africano. Esta fuente provee información rigurosa a nivel nacional sobre fecundidad, salud y mortalidad materno-infantil, entre otras muchas dimensiones para un total de 90 países en de desarrollo. ${ }^{3}$ En particular, son de interés el contexto socioeconómico, la religión y el peso al nacer de las personas.

Como primer paso se recopilaron los datos disponibles para África eliminando aquellas ondas que no incluyen información acerca de la religión de la madre, información fechas de nacimiento ${ }^{4}$, el peso al nacer y las medidas de altura y peso durante los primeros 5 años de vida del niño. Este criterio deja 86 muestras para 37 países africanos. Con el objetivo de crear el panel, y luego de normalizar la nomenclatura de las variables, se agregaron las bases de datos de cada onda y país. La base resultante permite observar los partos de las mujeres en los 5 años anteriores a la entrevista. ${ }^{5}$

En segundo lugar, se descartaron aquellas observaciones con problemas de coherencia por fechas de nacimiento o mediciones antropométricas incongruentes con las edades para evitar posibles sesgos de estimación provenientes de mal informe o medición o anormalidades genéticas.

Por otro lado, se examinaron variables z-scores reportadas, en particular Weight for age (Peso por edad) y Weight for height (Peso según altura) que están disponibles en la base para los niños. Puesto que las poblaciones de referencia son diferentes en diversas fases

\footnotetext{
${ }^{2}$ Debe tenerse en cuenta que la decisión de no realizar el ayuno es una decisión personal y esta correlacionada con el nivel de religiosidad del contexto de su lugar de origen.

3 Para el presente estudio se ha utilizado el peso al nacer como fuente de estudio por su precisión en la base de datos. Sin embargo, el mismo se podría extender a la altura u otras medidas antropométricas como por ejemplo el ancho del brazo del niño o diferentes medidas craneales.

${ }^{4}$ Del mismo modo que Karimi (2013) las fechas exactas de nacimiento dentro del mes fueron imputadas para un porcentaje de los datos. Este procedimiento fue realizado mediante una distribución uniforme entre los días múltiplos de 5 (corregidas por la característica del mes de febrero)

${ }^{5} \mathrm{Si}$ bien la configuración final de la base de datos no permite el completo estudio del continente, la variabilidad de población musulmana, ambientes socioeconómicos, y genética permite identificar el efecto deseado.
} 
de las DHS, niños con la misma edad y peso pueden tener valores que difieren entre muestras. Para solucionar este problema, se han tomado los Child Growth Standards provistos en 2006 por la Organización Mundial de la Salud para niños. Estas tienen la característica de diferenciarse por género a través de los meses hasta que el niño termina su cuarto año, específicamente hasta el mes 60 de vida. Luego de computar los Z-scores uniformes, se descartan aquellas observaciones que no se encuentren en el intervalo que va desde -6 hasta 6 .

Por último, se consideraron solo aquellas familias que incluyen reportes sobre la salud de los niños, educación de los padre y riqueza de los hogares. El número total de observaciones con información completa es de 264500 observaciones para 37 países. En la tabla 1 se reportan la cantidad de personas por país, pertenencia al islam y nivel de exposición al mes del Ramadán. Como se observa, la variedad de países es muy amplia en términos de cantidad de población encuestada y presencia de musulmanes. En algunos casos, como Marruecos y Comorros no se encuentran hogares no musulmanes, mientras que en Namibia, Lesoto, Sud África y Swaziland sucede lo contrario. Además se detectan gran cantidad de observaciones con exposición completa al Ramadán, en el islam y fuera. 
Tabla 1: Descripción de la Base de Datos

\begin{tabular}{|c|c|c|c|c|c|c|c|}
\hline & \multirow[b]{2}{*}{ Total de Observaciones } & \multicolumn{3}{|c|}{ Población Musulmana } & \multicolumn{3}{|c|}{ Población No Musulmana } \\
\hline & & No expuesto & Parcial & Exp. Total & No expuesto & Parcial & Exp. Total \\
\hline Burkina Faso & 16067 & 975 & 331 & 3051 & 2836 & 955 & 7919 \\
\hline Benin & 13525 & 510 & 144 & 1541 & 2474 & 894 & 7962 \\
\hline Burundi & 4699 & 45 & 11 & 159 & 1138 & 394 & 2952 \\
\hline Rep Dem del Congo & 6209 & 24 & 11 & 115 & 1141 & 414 & 4504 \\
\hline República Centroafricana & 1422 & 33 & 3 & 130 & 285 & 107 & 864 \\
\hline Congo (Brazzaville) & 4191 & 28 & 7 & 52 & 842 & 286 & 2976 \\
\hline Cote de Ivore & 3480 & 324 & 127 & 1057 & 443 & 150 & 1379 \\
\hline Camerún & 14898 & 450 & 170 & 1483 & 2662 & 1119 & 9014 \\
\hline Egipto & 13224 & 2902 & 991 & 8780 & 112 & 45 & 394 \\
\hline Etiopía & 2606 & 201 & 52 & 565 & 412 & 150 & 1226 \\
\hline Gabón & 3733 & 100 & 41 & 396 & 653 & 236 & 2307 \\
\hline Ghana & 3174 & 100 & 33 & 339 & 620 & 191 & 1891 \\
\hline Guinea & 4746 & 871 & 298 & 2808 & 146 & 50 & 573 \\
\hline Kenia & 9730 & 197 & 66 & 637 & 1982 & 605 & 6243 \\
\hline Comoras & 416 & 84 & 33 & 299 & 0 & 0 & 0 \\
\hline Liberia & 840 & 24 & 11 & 71 & 143 & 50 & 541 \\
\hline Lesoto & 5114 & 0 & 1 & 1 & 1326 & 439 & 3347 \\
\hline Marruecos & 3914 & 982 & 350 & 2582 & 0 & 0 & 0 \\
\hline Madagascar & 11071 & 39 & 15 & 97 & 2561 & 773 & 7586 \\
\hline Malí & 7479 & 1654 & 548 & 4822 & 97 & 30 & 328 \\
\hline Malawi & 23806 & 721 & 219 & 2035 & 4829 & 1672 & 14330 \\
\hline Mozambique & 7257 & 190 & 63 & 600 & 1505 & 501 & 4398 \\
\hline Nigeria & 6563 & 393 & 137 & 1365 & 1082 & 354 & 3232 \\
\hline Níger & 5241 & 1289 & 368 & 3503 & 21 & 5 & 55 \\
\hline Namibia & 8046 & 0 & 0 & 0 & 1877 & 631 & 5538 \\
\hline Ruanda & 12851 & 59 & 20 & 230 & 2758 & 998 & 8786 \\
\hline Sierra Leona & 2026 & 259 & 91 & 1099 & 113 & 32 & 432 \\
\hline Senegal & 14984 & 3495 & 1281 & 9691 & 125 & 41 & 351 \\
\hline Santo Tomé y Príncipe & 1554 & 3 & 2 & 7 & 346 & 105 & 1091 \\
\hline Swazilandia & 2340 & 0 & 0 & 1 & 540 & 172 & 1627 \\
\hline Chad & 1930 & 265 & 86 & 762 & 188 & 79 & 550 \\
\hline Togo & 1052 & 39 & 17 & 134 & 177 & 93 & 592 \\
\hline Tanzania & 12635 & 1240 & 400 & 3418 & 1688 & 692 & 5197 \\
\hline Uganda & 7156 & 322 & 82 & 865 & 1410 & 412 & 4065 \\
\hline Sudáfrica & 3369 & 0 & 0 & 0 & 795 & 273 & 2301 \\
\hline Zambia & 11410 & 9 & 3 & 28 & 2528 & 917 & 7925 \\
\hline Zimbabue & 11941 & 13 & 7 & 41 & 2856 & 859 & 8165 \\
\hline Toda la muestra & 264699 & 17840 & 6019 & 52764 & 42711 & 14724 & 130641 \\
\hline
\end{tabular}

Fuente: elaboración propia en base a las bases de datos DHS 


\subsection{Medidas de Exposición al Ramadán}

Se utiliza la fecha exacta de nacimiento y se compara con el día de inicio del mes del Ramadán anterior (tomando aproximadamente un año previo a la fecha de parto) para calcular el día de la gestación en el que comenzó la superposición analizada. El Ramadán dura 30 días y varía en el calendario solar estándar. Dada las ondas de estudio, debió contarse con los días de inicio desde 1980 hasta el presente para agregarse a la base. Para el análisis, se supone que la gestación tiene una duración estándar de 40 semanas o 280 días por lo que el trimestre y la cantidad de días puede determinarse con facilidad.

La configuración de la base de datos permite estimar la cantidad de horas de exposición intrauterina al Ramadán. Con este fin, se establecieron el o los meses exactos de coincidencia entre el embarazo y el Ramadán. Es decir, más allá del momento particular de desarrollo uterino, en cuál de los 365 días comenzó el ayuno. Debe notarse que como esta fecha no depende del mismo calendario, en la mayoría de las veces abarca días de diferentes meses. Luego, se multiplican por la cantidad de horas de luz solar (o heleofanía) según su latitud y mes. En caso de que sean dos meses, es necesario sumar ambos datos a fin de obtener la cantidad total de horas de exposición. Si el Ramadán ocurre al principio o al final de una gestación, la cantidad de días es menor a 30 pero el proceso es el mismo. En la base de datos del 78\% de los partos de madres musulmanas sufrieron algún tipo de superposición con el mes, lo que provee variabilidad.

Por último, se ha elegido trabajar con la exposición en términos de horas por trimestre o por mes de un modo similar al que lo hace Karimi (2014). Se asumirá 279 como el número total de días de desarrollo uterino. La ventaja principal es que es divisible por 3 y por 9 según si se quiere estudiar las horas de luz por trimestre o mes respectivamente. Por consiguiente, cada mes dura 31 días y cada trimestre 93.

En resumen, las variables de Horas de Exposición al Ramadán se midieron de la siguiente manera:

HLS $_{279}$ : Cantidad de Horas de Exposición al Ramadán durante toda la gestación

HLSti: Cantidad de Horas de Exposición al Ramadán durante el trimestre i, donde $\mathrm{i}=1,2,3$

HLS $_{\text {mi: }}$ Cantidad de Horas de Exposición al Ramadán durante el mes i, donde i=1,2..9 


\subsection{Descripción de los datos}

Para identificar el efecto del ayuno durante el Ramadán, se exploraron variaciones en la religión y en los diferentes niveles de superposición entre la gestación y el período de estudio. Se buscó, por ejemplo, identificar diferencias en el peso al nacer y la exposición entre musulmanes y no musulmanes. El principal objetivo es poner a prueba el supuesto de identificación mencionado anteriormente sobre la elección de la fecha de concepción.

En esta sección se presentan estadísticas descriptivas de las características observables de los niños, sus familiares y sus respectivos ambientes. A tal fin, los individuos fueron divididos entre musulmanes y no profesantes del islam. A su vez, cada grupo se clasificó en tres conjuntos diferentes: individuos que no han estado expuestos al Ramadán durante su propia gestación, aquellos expuestos parcialmente (1 a 29 días de la exposición), y los que han sido totalmente expuestos a este mes (30 días después de la exposición) durante su desarrollo fetal.

De este modo, la exposición al riego tiene dos aspectos principales. Por un lado, si la mujer estudiada profesa o no el Islam y por el otro, el grado de exposición al Ramadám. La cantidad de días en los que la mujer embarazada se enfrenta a desorden o ejerce el ayuno tiene diferentes efectos sobre el peso al nacer de los niños nacidos.

En la tabla 2 presenta la media y desvió estándar un grupo de variables representativas observables socio-ambientales de la población nacida en el lapso de los 5 años previos a que el hogar fuese encuestado. 
Tabla 2: Estadísticas descriptivas -Todos los Países

\begin{tabular}{|c|c|c|c|c|c|c|}
\hline & \multicolumn{3}{|c|}{ Población Musulmana } & \multicolumn{3}{|c|}{ Población No Musulmana } \\
\hline & No expuesto & Parcial & Exp. Total & No expuesto & Parcial & Exp. Total \\
\hline \multirow[t]{2}{*}{ Edad de las Madres } & 28.608 & 28.625 & 28.598 & 28.223 & 28.277 & 28.298 \\
\hline & $(6.667)$ & $(6.750)$ & $(6.722)$ & $(6.680)$ & (6.712) & (6.703) \\
\hline \multirow[t]{2}{*}{ Total de hijos } & 3.699 & 3.661 & 3.679 & 3.498 & 3.530 & 3.538 \\
\hline & (2.413) & (2.381) & $(2.402)$ & (2.306) & (2.317) & (2.326) \\
\hline \multirow[t]{2}{*}{ Ordén de Nacimiento } & 3.400 & 3.353 & 3.378 & 3.204 & 3.231 & 3.239 \\
\hline & $(2.391)$ & $(2.350)$ & $(2.379)$ & $(2.274)$ & $(2.280)$ & (2.291) \\
\hline \multirow[t]{2}{*}{ Años de Educación de las madres } & 4.169 & 4.143 & 4.165 & 5.751 & 5.769 & 5.766 \\
\hline & $(4.866)$ & $(4.838)$ & $(4.911)$ & (4.113) & (4.095) & (4.108) \\
\hline \multirow[t]{2}{*}{ Años de Educación de los padres } & 4.631 & 4.644 & 4.635 & 4.390 & 4.388 & 4.366 \\
\hline & (1.879) & $(1.860)$ & $(1.878)$ & (2.007) & (1.980) & (1.992) \\
\hline \multicolumn{7}{|l|}{ Porcentajes } \\
\hline Población Urbana & $57.735 \%$ & $57.352 \%$ & $56.794 \%$ & $38.725 \%$ & $39.466 \%$ & $39.859 \%$ \\
\hline Población Pobre & $24.243 \%$ & $23.708 \%$ & $24.714 \%$ & $44.497 \%$ & $43.541 \%$ & $43.949 \%$ \\
\hline Población Rica & $58.021 \%$ & $59.694 \%$ & $57.628 \%$ & $35.885 \%$ & $36.213 \%$ & $36.420 \%$ \\
\hline Población Promedio & $17.735 \%$ & $16.597 \%$ & $17.658 \%$ & $19.618 \%$ & $20.246 \%$ & $19.630 \%$ \\
\hline Mellizos & $4.344 \%$ & $4.253 \%$ & $3.861 \%$ & $3.414 \%$ & $3.898 \%$ & $3.701 \%$ \\
\hline
\end{tabular}

Fuente: elaboración propia en base a las bases de datos DHS

Nota: los desvios estándar se expresan en parénthesis. La educación del padre y de la madre están expresados en años. Además, el porcentaje de población pobre y rica fueron calculados a partir de los dos últimos y primeros quintiles de bienestar respectivamente

A fin de profundizar este estudio, en la tabla 3 se presentan los mismos estadísticos para los países con mayor porcentaje de individuos que profesan el Islam ${ }^{6}$. Este criterio deja a la base de datos con los 10 países de la comparación de los niños musulmanes expuestos y no expuestos en términos de las características familiares no muestra signos de sesgo de selección en la exposición al Ramadán en términos de promedios.

${ }^{6}$ El criterio utilizado es que por lo menos el 50\% de la población profese el Islam. 
Tabla 3: Estadísticas descriptivas -Países con mas de 50\% de población musulmana

\begin{tabular}{|c|c|c|c|c|c|c|}
\hline & \multicolumn{3}{|c|}{ Población Musulmana } & \multicolumn{3}{|c|}{ Población No Musulmana } \\
\hline & No expuesto & Parcial & Exp. Total & No expuesto & Parcial & Exp. Total \\
\hline \multirow[t]{2}{*}{ Edad de las Madres } & 28.133 & 28.182 & 28.185 & 28.005 & 28.119 & 28.084 \\
\hline & $(6.706)$ & $(6.785)$ & $(6.846)$ & $(6.707)$ & $(6.787)$ & (6.763) \\
\hline \multirow[t]{2}{*}{ Total de hijos } & 3.970 & 3.995 & 3.903 & 3.555 & 3.604 & 3.564 \\
\hline & $(2.425)$ & (2.473) & $(2.448)$ & $(2.255)$ & (2.289) & (2.288) \\
\hline \multirow[t]{2}{*}{ Ordén de Nacimiento } & 1.307 & 1.285 & 1.285 & 1.290 & 1.293 & 1.278 \\
\hline & $(0.538)$ & $(0.523)$ & $(0.520)$ & $(0.516)$ & $(0.526)$ & $(0.503)$ \\
\hline \multirow[t]{2}{*}{ Años de Educación de las madres } & 2.855 & 2.745 & 2.942 & 5.051 & 5.015 & 5.081 \\
\hline & (3.497) & (3.374) & $(3.525)$ & (3.913) & (3.940) & (3.933) \\
\hline \multirow[t]{2}{*}{ Años de Educación de los padres } & 4.378 & 4.644 & 4.386 & 4.369 & 4.334 & 4.334 \\
\hline & $(2.165)$ & $(2.111)$ & $(2.186)$ & (2.194) & $(2.125)$ & $(2.144)$ \\
\hline \multicolumn{7}{|l|}{ Porcentajes } \\
\hline Población Urbana & $44.694 \%$ & $45.597 \%$ & $45.959 \%$ & $34.799 \%$ & $35.679 \%$ & $37.084 \%$ \\
\hline Población Pobre & $44.643 \%$ & $43.711 \%$ & $43.136 \%$ & $47.302 \%$ & $47.773 \%$ & $46.599 \%$ \\
\hline Población Rica & $33.929 \%$ & $34.591 \%$ & $35.738 \%$ & $30.979 \%$ & $30.988 \%$ & $31.979 \%$ \\
\hline Población Promedio & $21.429 \%$ & $21.698 \%$ & $21.126 \%$ & $21.719 \%$ & $21.240 \%$ & $21.421 \%$ \\
\hline Mellizos & $5.510 \%$ & $4.088 \%$ & $4.593 \%$ & $4.182 \%$ & $4.627 \%$ & $4.091 \%$ \\
\hline
\end{tabular}

Fuente: elaboración propia en base a las bases de datos DHS

Nota: los desvios estándar se expresan en parénthesis. La educación del padre y de la madre están expresados en años. Además, el porcentaje de población pobre y rica fueron calculados a partir de los dos últimos y primeros quintiles de bienestar respectivamente

Este ejercicio se repite para tres variables relacionadas con la gestación del niño: la cantidad de horas de horas de exposición al Ramadán promedio durante el desarrollo, el peso al nacer y Weight-for-age Z-score de los niños. En este caso la tabla 4 provee las estimaciones para toda la muestra y para países con más del $10 \%$ y del $50 \%$ de población musulmana.

Como se observa, a medida que el tamaño de población de musulmanes aumente, se observa una pequeña diferencia en el peso al nacer. Además, la varianza de los pesos es mayor entre los musulmanes completamente expuestos al Ramadán que aquellos con superposición parcial. Esta diferencia representa aproximadamente entre un 5 y $10 \%$. 


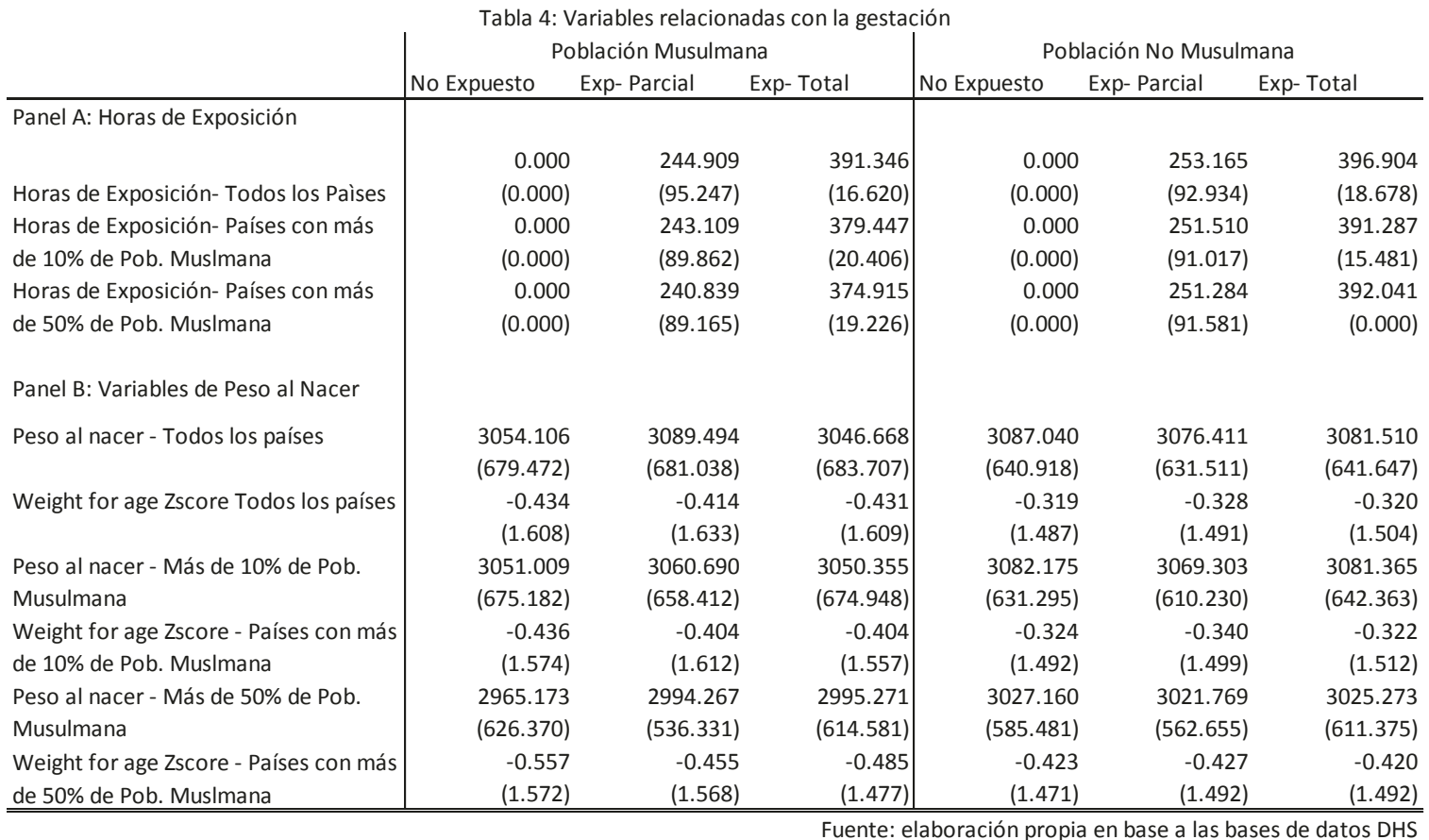
Nota: Los desvios estándar se expresan debajo de los promedios. Z-scores estimados con la metodología de OMS 2006

En la tabla 5, se exhiben los promedios (y sus respectivos desvíos estándar) de los pesos por semestre de edad hasta los 24 meses. Del mismo modo que sucede en el peso al nacer, aquellos niños musulmanes con exposición completa al Ramadán presentan desvíos de mayor magnitud en los desvíos estándar cuando la superposición es completa. Esto puede reflejar las variaciones en el comportamiento por parte de las madres.

Tabla 5: Pesos Promedio por semestre de Edad - Toda la muestra

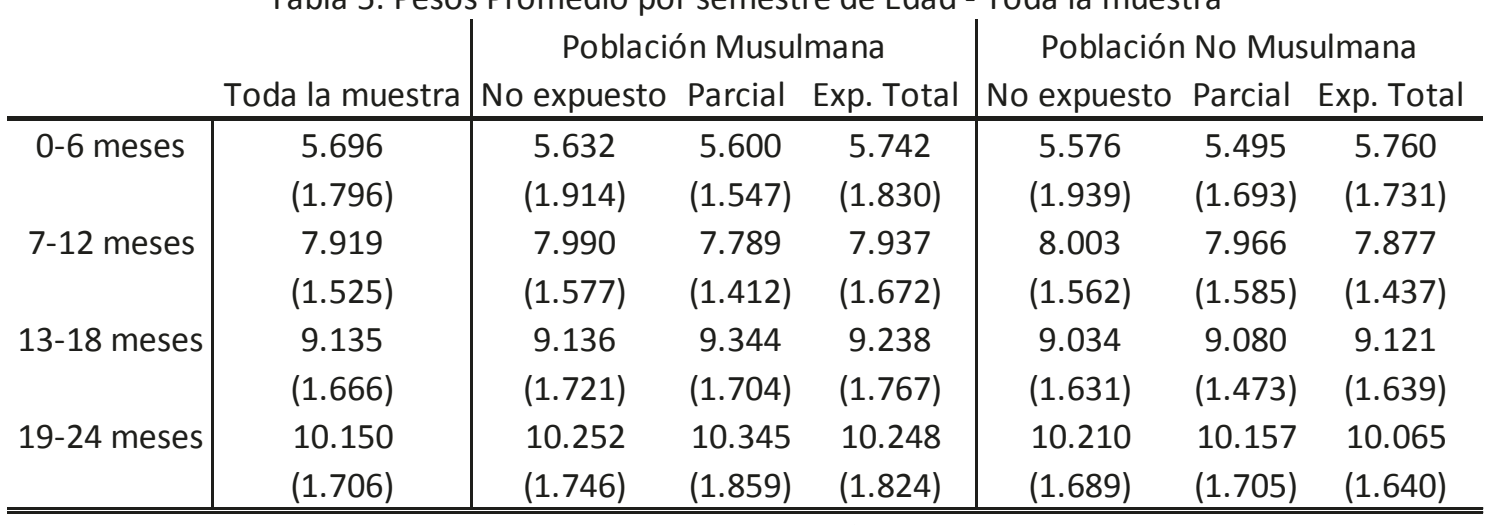

Fuente: elaboración propia en base a las bases de datos DHS Nota: los desvíos estándar se expresan en parentesis 


\section{Modelo Econométrico}

La primera aproximación a la base de datos, plantea la siguiente ecuación:

$$
\mathrm{y}_{\mathrm{itg}}=\theta_{\mathrm{itg}}+\mu_{\mathrm{s}}+\left(\mathrm{HLS}_{\mathrm{js}} \mathrm{xMuslim}{ }_{\mathrm{i}}\right) \boldsymbol{\beta}+\mathrm{X}_{\mathrm{itg}} \boldsymbol{\varphi}+\varepsilon_{\mathrm{itg}}
$$

donde $\mathrm{y}_{\text {itg }}$ puede ser cualquier variable del conjunto de medidas antropométricas que incluyen el peso al nacer y en el momento de la encuesta y las variables Z-scores calculadas según el criterio de la OMS de $2006^{7}$ para el niño i nacido en el momento $t$ dentro del paísg. Además, $\mathrm{HLS}_{\mathrm{js}}$ es la variable o el conjunto de variables de horas de exposición al Ramadán como fue explicada en la sección anterior. Es decir, la exposición total HLS279, por trimestre $\left\{\mathrm{HLS}_{\mathrm{t} 1} \mathrm{HLS}_{\mathrm{t} 2} \mathrm{HLS}_{\mathrm{t} 3}\right\}$ o mensuales $\left\{\mathrm{HLS}_{\mathrm{m} 1}\right.$, $\mathrm{HLS}_{\mathrm{m} 2 \ldots} \ldots$ HLSm9\}. Esta variable se multiplica por una variable categórica $\gamma_{\mathrm{i}}$ asociada con el parámetro de profesión de la religión musulmana Muslim $_{\mathrm{i}}$, que toma valor $1 \mathrm{si}$ esta familia profesa el Islam. $\beta$ es el vector de parámetros asociados a este efecto. La letra $\theta$ representa a la constante del modelo.

La letra $\mu$ (con su respectivo subíndice s) representa un efecto fijo por madre que se debe agregar en el modelo porque es posible que su tamaño y estilo de vida también determinen el peso del bebé al nacer y su decisión de llevar adelante el ayuno o no. Idealmente, los investigadores que abordan el tema preferirían comparar las medidas antropométricas de niños cuyas madres profesantes del Islam fueran asignadas de manera aleatoria a ayunar durante el Ramadán con las de niños que hubiesen recibido el tratamiento contrario. Este procedimiento brindaría estimadores consistentes. Sin embrago, no es posible llevar adelante este tipo de estudios controlados. Es por esta razón que es muy importante tener en cuenta las características de las madres. Si se supone por ejemplo, que el nivel educativo de la madre se encuentra negativamente correlacionado con su elección de ayunar y positivamente correlacionado con la calidad de la nutrición que su hijo recibe, puede darse que mala nutrición (en lugar del comportamiento asociado al Ramadán) afecten de manera negativa en los resultados de corto y mediano plazo luego del nacimiento. Por consiguiente, los resultados adversos

\footnotetext{
${ }^{7}$ Estos estándares son internacionalmente representativos y reemplazan a los criterios NCHS utilizados anteriormente. Las normas de la OMS son también más exactos, especialmente en la primera infancia. Las normas están disponibles públicamente en http://www.who.int/nutgrowthdb/en/
} 
adjudicados al mes sagrado, se deban en realidad parcialmente a que niños hijos de madres menos educadas reciben menos calidad en su nutrición.

Por su parte, $\mathrm{X}_{\text {itg }}$ es la fila de la matriz de incidencia que asocia los efectos de un grupo de variables socioeconómicas para la observación itg, con el vector paramétrico $\boldsymbol{\varphi}$. Por último, $\varepsilon_{\text {itg }}$ es el error del modelo. Su objetivo es controlar por el ambiente y el bienestar. Tomando a Karimi (2014), se armó un Índice de Bienestar mediante el método de componentes principales que se utilizó como control para las estimaciones. La lista utilizada para obtener el indice incluye a la fuente de agua potable, el tipo de baño, el principal material del piso de la casa, el acceso a la electricidad y posesión de radio y de televisión. Una vez extraídos los componentes se armó un índice cualitativo a partir de los quintiles de la distribución donde 1 equivale a muy pobre, 2 a pobre, 3 a promedio, 4 a rico y 5 a muy rico. Acompañan a esta variable de control dos de vital importancia: la educación del padre y el orden de nacimiento en la familia. Asimismo, se consideró una variable cualitativa de si el niño es hombre o mujer por las diferencias que puedan generarse a partir de este factor.

Cabe señalar que la no inclusión de las variables puede tener un efecto negativo en el modelo por la precisión de los estimadores y por la posibilidad de no captar el efecto deseado. En otras palabras, de no ser tomadas en cuenta estas variables de control, es probable que no se pueda establecer el efecto del peso al nacer, sino que en realidad se estén observando las consecuencias de otras variables. La lista puede incluir cuestiones socioeconómicas (por ejemplo, la educación de los miembros de su ambiente) que también afectan la decisión de ayunar de la madres o los respectivos pesos al nacer de sus hijos.

Cuando las variables independientes comparan a niños de diferentes edades, se incluyen además, variables cualitativas por semestre de edad. En modelos con niños de hasta 5 años, el número de variables sería 10: de 0 a 6 meses, de 7 a 12 meses, hasta llegar a los 54 a 60 meses de vida. 


\section{Resultados principales}

En esta sección se presentan los resultados de las estimaciones realizadas para el primer modelo. En primer lugar, se estimaron los modelos de peso al nacer de los niños nacidos (tanto los nacimientos que resultaron en un niño sano como los que resultaron en fallecimiento) teniendo en cuenta el efecto fijo por madre y diversas especificaciones que toman en cuenta las horas de exposición al Ramadán, el efecto fijo por madre y los controles sociales y ambientales apropiados para cada estimación. A su vez, se practicaron estimaciones con diferentes tamaños de muestra, según la cantidad de musulmanes que habiten en los países: para toda la muestra, para países con más del $10 \%$ de población musulmana y para países del 50\%. La segmentación se realizó para captar variaciones en el comportamiento de los individuos, es más probable que en países en donde el porcentaje de musulmanes es mayor, el grado de ayuno sea mayor. Por otro lado, se realizan estimaciones para varios números de días de exposición al Ramadán. Así como lo hace Karimi (2014), este estudio pone a prueba el hecho de que el efecto de ayunar aparece luego de 10 días de confluencia entre el embarazo y el Ramadán.

\subsection{Peso al nacer}

En la tabla 6 se exponen los resultados para el peso al nacer. Se presentan cuatro especificaciones. Las dos primeras no limitan en la cantidad de observaciones según nivel de musulmanes que habitan en los países o días de solapa. Con el fin de mantener la consistencia de los resultados y evitar errores de medición, la estimación se limita a las observaciones cuyo peso estaba escrito en su registro médico personal. Además, este tendrá el beneficio de evitar observaciones auto-reportadas. Luego se agrega el índice de Bienestar construido y las variables de control mencionadas en la especificación del modelo para ciertas especificaciones. 
Tabla 6: Estimaciones Para el peso al nacer

\begin{tabular}{|c|c|c|c|c|c|c|}
\hline VARIABLES & $\begin{array}{c}\text { Toda la } \\
\text { (1) } \\
\text { S/Controles }\end{array}$ & $\begin{array}{l}\text { Dlación } \\
\qquad(2) \\
\text { C/Controles }\end{array}$ & $\begin{array}{c}\text { Más de } 10 \% \\
(3) \\
\text { S/Controles }\end{array}$ & $\begin{array}{c}\text { Pob. Musulmana } \\
(4) \\
\text { C/Controles } \\
\end{array}$ & $\begin{array}{c}\text { Más de } 50 \% \\
\text { (5) } \\
\text { S/Controles }\end{array}$ & $\begin{array}{c}\text { Pob. Musulmana } \\
(6) \\
\text { C/Controles } \\
\end{array}$ \\
\hline Hs Luz 3er. Tr X Musulman (=1) & $\begin{array}{l}-0.7241^{*} \\
{[0.3787]}\end{array}$ & $\begin{array}{c}-0.8855^{\star *} \\
{[0.4265]}\end{array}$ & $\begin{array}{l}-1.0272^{*} \\
{[0.5552]}\end{array}$ & $\begin{array}{c}-1.0727^{*} \\
{[0.6081]}\end{array}$ & $\begin{array}{c}-0.5897^{* *} \\
{[0.1935]}\end{array}$ & $\begin{array}{c}-0.7188^{\star *} \\
{[0.2414]}\end{array}$ \\
\hline Hs Luz 2er. Tr X Musulman (=1) & $\begin{array}{c}-0.7341^{* *} \\
{[0.3357]}\end{array}$ & $\begin{array}{c}-0.7161^{\star *} \\
{[0.3361]}\end{array}$ & $\begin{array}{c}-0.9346 * \\
{[0.4688]}\end{array}$ & $\begin{array}{c}-0.9886^{*} \\
{[0.4897]}\end{array}$ & $\begin{array}{l}-0.8272^{*} \\
{[0.4357]}\end{array}$ & $\begin{array}{c}-1.0532^{* *} \\
{[0.3679]}\end{array}$ \\
\hline Hs Luz 1er Tr. X Musulman (=1) & $\begin{array}{c}0.2598 \\
{[0.3913]}\end{array}$ & $\begin{array}{c}0.2394 \\
{[0.4062]}\end{array}$ & $\begin{array}{c}-0.09 \\
{[0.4558]}\end{array}$ & $\begin{array}{c}-0.155 \\
{[0.4430]}\end{array}$ & $\begin{array}{c}-0.1284 \\
{[1.0685]}\end{array}$ & $\begin{array}{c}-0.301 \\
{[0.9915]}\end{array}$ \\
\hline $\begin{array}{l}\text { Número de Observaciones } \\
\text { R-cuadrado } \\
\text { Efecto Fijo por Madre }\end{array}$ & $\begin{array}{l}28,921 \\
0.0142 \\
\mathrm{Si}\end{array}$ & $\begin{array}{l}28,899 \\
0.0295 \\
\mathrm{Si}\end{array}$ & $\begin{array}{l}20,028 \\
0.0126 \\
\mathrm{Si}\end{array}$ & $\begin{array}{l}20,013 \\
0.0266 \\
\mathrm{Si}\end{array}$ & $\begin{array}{l}11,560 \\
0.0049 \\
\mathrm{Si}\end{array}$ & $\begin{array}{l}11,554 \\
0.0251 \\
\mathrm{Si}\end{array}$ \\
\hline
\end{tabular}

Nota: Los desvios estándar se expresan entre paréntesis. ${ }^{* *} p<0.01,{ }^{* *} p<0.05,{ }^{*} p<0$. Los desvios fueron clusterados a nivel de País

Si bien pequeño, se observa un efecto significativo y adverso en el peso al nacer cuando el Ramadán ocurrió en el segundo o tercer trimestre de la gestación. Este sobrevive a las tres niveles de estudio. En promedio el Ramadán tiene una duración de 11 horas por día. Esto implica que el efecto de la mala nutrición o el desorden ambiental tiene un efecto aproximado de entre 6 gr y 10 gr. Por otro lado, se observa que el efecto en el primer trimestre no resulta significativo. ${ }^{8}$

En la tabla 7 se presentan las estimaciones para Z-scores Weight for age para los coeficientes de las Horas de luz solar por trimestre para toda la población, y para los países con más del 50\% de población musulmana.

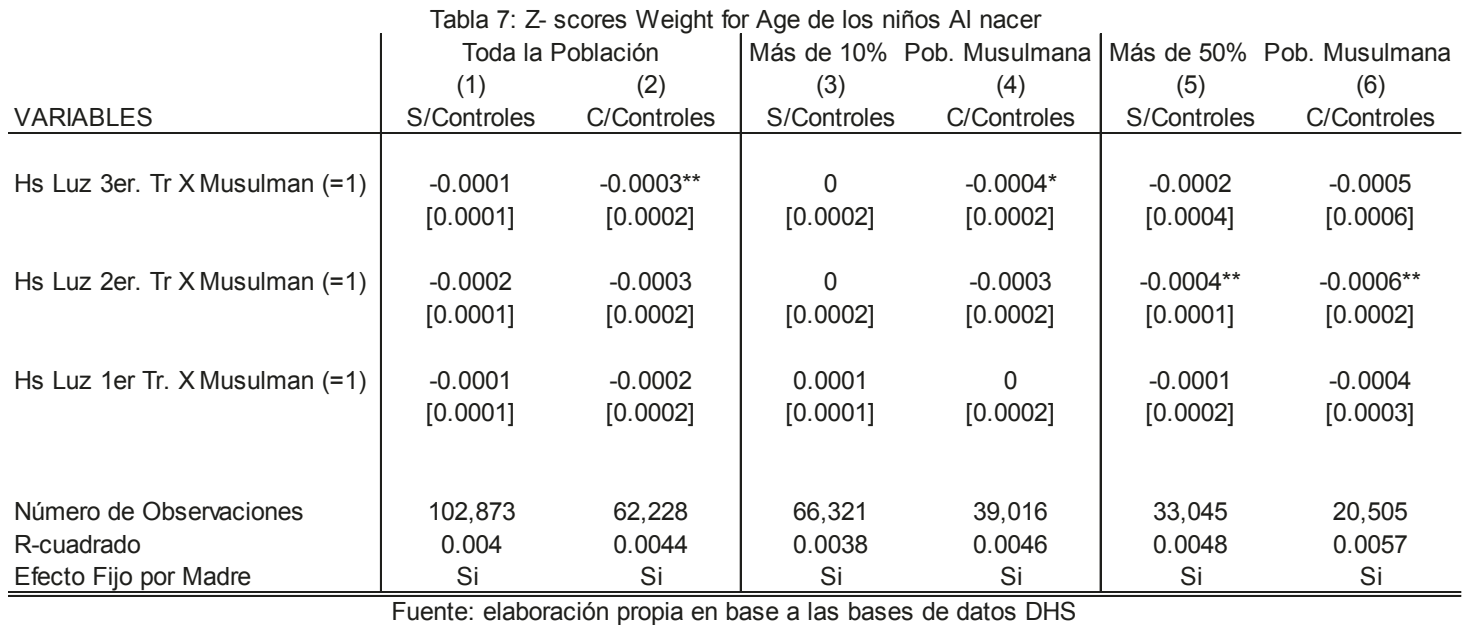

Nota: Los desvios estándar se expresan entre paréntesis. ${ }^{* *} p<0.01,{ }^{* *} p<0.05,{ }^{*} p<0$. Los desvios fueron clusterados a nivel de

\footnotetext{
${ }^{8}$ Se realizó una estimación por separado en para todos los países (sin importar la cantidad de musulmanes) en la que se incluye la educación de la madre. Resulta interesante que esta variable es positiva y significativa. Su coeficiente equivale a 253.7 gr. Lo que señala aún más, la importancia de tener información acerca del comportamiento desordenado.
} 
En este caso, las estimaciones muestran un impacto pequeño pero significativo para los desvíos estándar. Se halló un efecto en el segundo trimestre para los países con más de $50 \%$ de musulmanes. Con el fin de hacer más claro el análisis y obtener el impacto de una exposición total al Ramadán, se multiplican los resultados por 330 tal como lo hizo Karimi (2014). ${ }^{9}$ Esta simple cuenta brinda una desviación estándar promedio de entre un 0.13 y un 0.20 con respecto al criterio de la OMS de 2006.

\section{a. Especificación alternativa}

En la tabla 8 a continuación se exhiben las estimaciones para las Horas de luz solar por trimestre para toda la población para los países y con más del $10 \%$ de población musulmana respectivamente.

En ella se observa que los efectos del ayuno o el desorden siguen siendo significativos a un nivel del 5\%. A partir del segundo trimestre, la magnitud puede incluso llegar a los 12 gr por día en promedio. Mientras que para los primeros meses, no se encontraron impactos en los coeficientes, si se percibe que el efecto está presente con a partir del cuarto mes de gestación. Este hecho consistente con lo encontrado en la sección anterior.

Por último, los desvíos estándar son similares a los encontrados en el modelo con Horas de Luz por trimestre.

\footnotetext{
${ }^{9}$ La cuenta es la siguiente: $30 \times 11=330$. El 11proviene de la media de horas de luz solar para todos los países y el 30 es explicado por la cantidad de días que dura el Ramadán.
} 
Tabla 8: Estimaciones para pesos para el peso al nacer por mes

(1)

Toda la Población
(2) Más de 10\% Pob. Musulmana

\section{VARIABLES}

Hs Luz 9no. Tr X Musulman (=1)

Hs Luz 8vo. Tr X Musulman (=1)

Hs Luz 7mo. Tr. X Musulman (=1)

Hs Luz 6to. Tr X Musulman (=1)

Hs Luz 5to. Tr X Musulman (=1)

Hs Luz 4to. Tr. X Musulman (=1)

Hs Luz 3er. Tr X Musulman (=1)

Hs Luz 2er. Tr X Musulman (=1)

Hs Luz 1er Tr. XMusulman (=1)

Número de Observaciones

R-cuadrado
$-0.4736$

[0.8944]

$-0.7664^{*}$

[0.4267]

$-0.63$

[0.5025]

$-0.9836^{*}$

[0.5657]

$0.7440^{* *}$

[0.3109]

$-0.9009^{* * *}$

[0.3262]

0.2211

[0.5032]

0.1153

[0.8993]

0.5178

[0.6504]

28,921

0.0224

\section{$-2.0458^{\star}$}

[1.0992]

$-1.4269^{* *}$

[0.6667]

$-1.3681$

[1.2815]

$-2.0218^{* * *}$

[0.3383]

$0.4505^{\star * *}$

[0.1420]

$-0.4331$

[0.6302]

$-0.5906$

[0.5357]

1.7680 ***

[0.3502]

$-1.0488$

[0.6177]

Nota: Los desvios estándar se expresan entre paréntesis. ${ }^{* * *} p<0.01,{ }^{* *} p<0.05,{ }^{*} p<0$. Los desvios fueron clusterados a nivel de País.

\subsection{Peso de los niños de hasta dos años}

La tabla 9 contiene los resultados de las estimaciones para los niños de hasta 24 meses con por lo menos 10 días de superposición entre su gestación y el Ramadán. Las medidas de exposición al Ramadán se calculan por trimestre aproximado de gestación. Además, al realizar una comparación entre individuos con diferentes edades, todas las variantes incluyen variables cualitativas indicadoras del semestre de edad. 


\begin{tabular}{|c|c|c|c|c|c|c|}
\hline VARIABLES & $\begin{array}{l}\text { Toda la } \\
\text { (1) } \\
\text { S/Controles } \\
\end{array}$ & $\begin{array}{l}\text { blación } \\
\text { (2) } \\
\text { C/Controles } \\
\end{array}$ & $\begin{array}{c}\text { Más de } 10 \% \\
(3) \\
\text { S/Controles }\end{array}$ & $\begin{array}{c}\text { Pob. Musulmana } \\
(4) \\
\text { C/Controles }\end{array}$ & $\begin{array}{c}\text { Más de } 50 \% \\
(5) \\
\text { S/Controles } \\
\end{array}$ & $\begin{array}{c}\text { Pob. Musulmana } \\
(6) \\
\text { C/Controles }\end{array}$ \\
\hline Hs Luz 3er. Tr X Musulman (=1) & $\begin{array}{l}-1.9866^{*} \\
{[1.1111]}\end{array}$ & $\begin{array}{l}-1.6057 \\
{[1.0860]}\end{array}$ & $\begin{array}{l}-3.1821 \\
{[2.1216]}\end{array}$ & $\begin{array}{l}-0.2718 \\
{[0.3574]}\end{array}$ & $\begin{array}{c}-6.8840^{* * *} \\
{[1.9452]}\end{array}$ & $\begin{array}{l}-7.6791^{* *} \\
{[2.6829]}\end{array}$ \\
\hline Hs Luz 2er. Tr X Musulman (=1) & $\begin{array}{l}-1.5577 \\
{[1.2132]}\end{array}$ & $\begin{array}{l}-1.3832 \\
{[1.2689]}\end{array}$ & $\begin{array}{c}-2.589 \\
{[2.6680]}\end{array}$ & $\begin{array}{l}-0.0337 \\
{[0.3578]}\end{array}$ & $\begin{array}{c}-7.5012^{* * *} \\
{[1.3109]}\end{array}$ & $\begin{array}{c}-8.0409^{* * *} \\
{[1.7578]}\end{array}$ \\
\hline Hs Luz 1er Tr. X Musulman (=1) & $\begin{array}{l}-1.0906 \\
{[1.0322]}\end{array}$ & $\begin{array}{c}-0.821 \\
{[1.2440]}\end{array}$ & $\begin{array}{l}-1.4558 \\
{[2.3254]}\end{array}$ & $\begin{array}{l}-0.7035^{\star *} \\
{[0.2854]}\end{array}$ & $\begin{array}{c}-5.5796^{* * *} \\
{[0.7421]}\end{array}$ & $\begin{array}{c}-6.5371^{* * *} \\
{[1.0958]}\end{array}$ \\
\hline $\begin{array}{l}\text { Número de Observaciones } \\
\text { R-cuadrado }\end{array}$ & $\begin{array}{l}65,900 \\
0.7773\end{array}$ & $\begin{array}{l}57,753 \\
0.7822\end{array}$ & $\begin{array}{l}41,444 \\
0.7815\end{array}$ & $\begin{array}{l}35,456 \\
0.7841\end{array}$ & $\begin{array}{c}18679.00 \\
0.8004\end{array}$ & $\begin{array}{l}15,156 \\
0.8032\end{array}$ \\
\hline
\end{tabular}

Nota: Los desvios estándar se expresan entre paréntesis. ${ }^{* *} p<0.01,{ }^{* *} p<0.05,{ }^{*} p<0$. Los desvios fueron clusterados a nivel de

El efecto comienza a ser significativo en la segunda especificación para el primer trimestre de gestación y es negativo. Es decir que por hora de ayuno, se espera que el niño nacido tenga, 0,7 gramos menos de peso. Considerando que el ayuno durante el Ramadán dura aproximadamente 11 horas, el ayuno materno tiene en promedio un efecto de 7,7 gramos por día. No obstante, que sea significativo en el primer trimestre es congruente con un efecto sobre el desarrollo de los órganos y un efecto a mediano plazo.

Cuando la especificación solo incluye a países con más del 50\% de población musulmana, el efecto se vuelve más significativo puesto que es más probable que estas mujeres practiquen el ayuno o convivan con familiares o amigos que se encuentre realizando este comportamiento.

Por otro lado, la tabla 10 contiene los resultados de Z-scores Weight for age y Weight for height. Para ambas variables, se presentan las estimaciones de los coeficientes de las Horas de luz solar por trimestre para toda la población y para los países con más del $50 \%$ de población musulmana. 


\begin{tabular}{|c|c|c|c|c|c|c|c|c|}
\hline \multirow[b]{2}{*}{ VARIABLES } & $\begin{array}{c}(1) \\
\text { Weight for }\end{array}$ & $\begin{array}{l}\text { Toda la P } \\
\text { (2) } \\
\text { Age Z-score }\end{array}$ & $\begin{array}{l}\text { oblación } \\
\text { (3) } \\
\text { Weight for h }\end{array}$ & $\begin{array}{l}(4) \\
\text { eight Z-score }\end{array}$ & $\begin{array}{c}\text { (1) } \\
\text { Weight for }\end{array}$ & $\begin{array}{c}\text { lás de } 50 \% \\
\text { (2) } \\
\text { Age Z-score }\end{array}$ & $\begin{array}{l}\text { ob. Musulma } \\
\text { (3) } \\
\text { Weight for h }\end{array}$ & $\begin{array}{l}\text { ha } \\
\text { eight Z-score }\end{array}$ \\
\hline & S/Controles & $\mathrm{C} /$ Controles & S/Controles & $\mathrm{C} /$ Controles & S/Controles & $\mathrm{C} /$ Controles & S/Controles & $\mathrm{C} /$ Controles \\
\hline Hs Luz 3er. Tr X Musulman & $\begin{array}{c}-0.0005 \\
{[0.0009]}\end{array}$ & $\begin{array}{c}-0.0006 \\
{[0.0009]}\end{array}$ & $\begin{array}{c}-0.0012 \\
{[0.0011]}\end{array}$ & $\begin{array}{c}-0.0014 \\
{[0.0012]}\end{array}$ & $\begin{array}{c}-0.0037 \\
{[0.0031]}\end{array}$ & $\begin{array}{l}-0.0069^{*} \\
{[0.0036]}\end{array}$ & $\begin{array}{l}-0.0046^{*} \\
{[0.0022]}\end{array}$ & $\begin{array}{c}-0.0088^{* *} \\
{[0.0026]}\end{array}$ \\
\hline Hs Luz 2er. Tr X Musulman & $\begin{array}{c}-0.0001 \\
{[0.0011]}\end{array}$ & $\begin{array}{c}-0.0001 \\
{[0.0010]}\end{array}$ & $\begin{array}{c}-0.0009 \\
{[0.0012]}\end{array}$ & $\begin{array}{c}-0.0009 \\
{[0.0012]}\end{array}$ & $\begin{array}{c}-0.0032 \\
{[0.0026]}\end{array}$ & $\begin{array}{l}-0.0057^{*} \\
{[0.0028]}\end{array}$ & $\begin{array}{c}-0.002 \\
{[0.0015]}\end{array}$ & $\begin{array}{l}-0.0048^{*} \\
{[0.0021]}\end{array}$ \\
\hline Hs Luz 1er Tr. X Musulman & $\begin{array}{c}0.0002 \\
{[0.0011]}\end{array}$ & $\begin{array}{c}0.0001 \\
{[0.0010]}\end{array}$ & $\begin{array}{c}-0.0004 \\
{[0.0011]}\end{array}$ & $\begin{array}{c}-0.0003 \\
{[0.0011]}\end{array}$ & $\begin{array}{c}-0.003 \\
{[0.0032]}\end{array}$ & $\begin{array}{c}-0.0054 \\
{[0.0030]}\end{array}$ & $\begin{array}{c}-0.0031 \\
{[0.0026]}\end{array}$ & $\begin{array}{l}-0.0055^{*} \\
{[0.0024]}\end{array}$ \\
\hline $\begin{array}{l}\text { Número de Observaciones } \\
\text { R-cuadrado }\end{array}$ & $\begin{array}{l}65,540 \\
0.0195\end{array}$ & $\begin{array}{l}57,431 \\
0.0497\end{array}$ & $\begin{array}{l}62,847 \\
0.0167\end{array}$ & $\begin{array}{l}55,026 \\
0.0213\end{array}$ & $\begin{array}{l}18,592 \\
0.0137\end{array}$ & $\begin{array}{l}15,084 \\
0.0529\end{array}$ & $\begin{array}{l}17,825 \\
0.0211\end{array}$ & $\begin{array}{l}14,429 \\
0.0573\end{array}$ \\
\hline
\end{tabular}

No se observan efectos en toda la población pero si en aquellos países más musulmanes del continente. Si bien son significativos al 5\%, estos son pequeños en términos de magnitud. El impacto del Ramadán es pequeño en los dos primeros años de vida del niño.

\subsection{Discusión}

El presente estudio buscó cuantificar el impacto de realizar ayuno (o al menos tener un comportamiento desordenado) durante el embarazo sobre el peso al nacer y los primeros semestres de vida. A través de diferentes composiciones de la base de datos, se encontró un efecto promedio de ayunar en el segundo o tercer trimestre que va entre 6 y $10 \mathrm{gr}$ para el peso al nacer de los niños. Si se considera que el Ramadán dura 30 días, el efecto promedio para un niño cuya gestación confluyó con el ayuno todo el período puede llegar a ser de hasta 300 gr. Esta cantidad representa 0,52 desvíos estándar de la media de la muestra. El efecto persiste incluso cuando se evalúa la hipótesis elegida teniendo en cuenta las Horas de Luz Solar a nivel mensual como variable explicativa, en lugar de observarlas por trimestre puesto que dicho efecto se observó a partir del cuarto mes.

Como fue tratado en varias secciones previas, este trabajo se enmarca en la literatura del Ramadán y del déficit alimentario en el desarrollo intrauterino por lo que es posible establecer cierta escala en los resultados previos. En primer lugar, Camacho (2008) observó una reducción de 11.6 gramos como producto de la exposición intrauterina al estrés provocado por explosiones aleatorias en Colombia. Almond y Mazunder (2011) encontraron un efecto de ayunar durante todo el primer mes gestación algo menor al analizado en el presente estudio. La estimación se encontró entre 40 y 51 gr en 
promedio de peso al nacer para Uganda y Michigan. Mientras tanto, en el trabajo de Majid (2012) se observó un efecto muy similar al encontrado en el presente trabajo: 270 grs. en Indonesia.

Es importante destacar que se advierte una relación entre el ayuno y el Ramadán persiste por lo menos hasta en los 24 meses de vida. Cuando la muestra toma en cuenta a países con más de 10\% de comunidad musulmana, se observó que el haber ayunado tuvo un efecto más adverso durante el primer trimestre de gestación intrauterina que en los subsiguientes. En este sentido, la magnitud de los efectos (tanto en términos de gramos como de variables Z-scores) aumentó en la especificación que solo incluye a los países con mayoría de población musulmana. Probablemente las mujeres que viven en estos países se vean más afectadas por el desorden social que cuando el Islam constituye una minoría. Este resultado sugiere que los costos relacionados con el cuidado de la salud perduran más allá de los primeros meses de vida.

Para el análisis realizado en esta tesis se empleó toda la base de datos, es decir, no se impusieron restricciones sobre la cantidad de días de confluencia entre el Ramadán y el desarrollo intrauterino. Estudios previos de ayuno durante el Ramadán limitan el análisis a aquellos individuos con exposición elevada, por ejemplo Almond y Mazunder (2011) emplearon datos de madres únicamente con los 30 días de superposición a lo largo de los 9 meses, y Karimi (2014) encontró efectos de ayunar sobre la altura de los niños y de las madres, a partir de 10 días de dicho comportamiento.

Otra cuestión de interés es aquella relacionada con la ausencia de efectos significativos para el peso al nacer por haber ayunado durante el primer trimestre. Este resultado es diferente de Almond y Mazunder (2011) puesto que en este trabajo se observó que el efecto de ayunar podía ser identificado únicamente en este trimestre. Sin embargo, otros trabajos, como el de Majid (2011) y Karimi (2014) informaron la presencia de efectos del ayuno en cualquier momento del embarazo, y en particular durante el segundo trimestre. 


\section{Chequeos de Robustez}

A modo de evaluar la calidad de los resultados encontrados, en esta sección se proponen especificaciones alternativas.

En primer lugar, el efecto sobre el peso estará sesgado si los padres establecen el embarazo con respecto a la fecha probable del Ramadán por lo que se chequeó si efecto adverso persistía bajo otra especificación en la que no se incluye el efecto fijo por madre. Este fue calculado del siguiente modo. Primero se limitó el estudio a madres con más de dos hijos cuyo primer niño fe expuesto al Ramadán pero su segundo no. Esto deja un total de 8232 casos en toda la muestra. Luego se creó la variable de la diferencia de pesos al nacer que será regresada en las variables de exposición al Ramadán del primer niño o niña. Los resultados se muestran en la tabla 11 a continuación

Tabla 11: Diferencia de Pesos al Nacer según exposición al Ramadán Modelos Sin Controles Modelos Con controles

Modelos de Diferencias Modelos de Diferencias Primer Hijo Expuesto Primer Hijo Expuesto VARIABLES PES(1er.) - PES (2do) PES(1er.) - PES (2do)

\begin{tabular}{|c|c|c|}
\hline Hs Luz 1er. Tr X Musulman (=1) & $\begin{array}{c}-0.033 \\
{[0.096]}\end{array}$ & $\begin{array}{c}-0.041 \\
{[0.098]}\end{array}$ \\
\hline Hs Luz 2er. Tr X Musulman (=1) & $\begin{array}{l}-0.222^{*} \\
{[0.111]}\end{array}$ & $\begin{array}{l}-0.232^{\star} \\
{[0.114]}\end{array}$ \\
\hline Hs Luz 3er Tr. X Musulman (=1) & $\begin{array}{c}-0.128 \\
{[0.089]}\end{array}$ & $\begin{array}{l}-0.142 \\
{[0.085]}\end{array}$ \\
\hline Observaciones & 8,232 & 8,013 \\
\hline $\mathrm{R}$ cuadrado & 0.017 & 0.018 \\
\hline Efecto Fijo por País & $\mathrm{Si}$ & $\mathrm{Si}$ \\
\hline
\end{tabular}

Se percibe un efecto pequeño pero significativo en la diferencia de pesos al nacer (expresada en gramos) de aquellas madres que poseen un hijo expuesto y otro no por lo que el impacto persiste a esta especificación.

Alternativamente, la tabla 12 a continuación contiene los coeficientes para las interacciones entre las Horas de Luz solar por trimestre y la variable de Hombre. En la 
parte inferior se presenta el estadístico t. Como se puede apreciar, no es posible rechazar la hipótesis de la significatividad estadística conjunta de los coeficientes en ninguna de las variantes del modelo.

\begin{tabular}{|c|c|c|c|c|c|c|}
\hline VARIABLES & $\begin{array}{l}\text { Toda la } \\
\text { (1) } \\
\text { S/Controles } \\
\end{array}$ & $\begin{array}{l}\text { blación } \\
\text { (2) } \\
\text { C/Controles }\end{array}$ & $\begin{array}{c}\text { Más de } 10 \% \\
(3) \\
\text { S/Controles }\end{array}$ & $\begin{array}{c}\text { Pob. Musulmana } \\
(4) \\
\text { C/Controles } \\
\end{array}$ & $\begin{array}{c}\text { Más de } 50 \% \\
(5) \\
\text { S/Controles }\end{array}$ & $\begin{array}{c}\text { Pob. Musulmana } \\
(6) \\
\text { C/Controles }\end{array}$ \\
\hline Hs Luz 3er. Tr X Musulman (=1) X Hombre(=1) & $\begin{array}{l}-0.246 \\
{[0.407]}\end{array}$ & $\begin{array}{l}-0.175 \\
{[0.339]}\end{array}$ & $\begin{array}{l}-0.618 \\
{[0.623]}\end{array}$ & $\begin{array}{c}-0.4 \\
{[0.473]}\end{array}$ & $\begin{array}{c}-0.677 \\
{[0.457]}\end{array}$ & $\begin{array}{l}-0.677 \\
{[0.457]}\end{array}$ \\
\hline Hs Luz 2er. Tr X Musulman (=1) X Hombre(=1) & $\begin{array}{l}-0.062 \\
{[0.284]}\end{array}$ & $\begin{array}{l}-0.058 \\
{[0.284]}\end{array}$ & $\begin{array}{l}-0.003 \\
{[0.408]}\end{array}$ & $\begin{array}{l}-0.001 \\
{[0.408]}\end{array}$ & $\begin{array}{l}-0.187 \\
{[0.729]}\end{array}$ & $\begin{array}{l}-0.187 \\
{[0.729]}\end{array}$ \\
\hline Hs Luz 1er Tr. X Musulman (=1) X Hombre(=1) & $\begin{array}{c}0.065 \\
{[0.258]}\end{array}$ & $\begin{array}{c}0.07 \\
{[0.259]}\end{array}$ & $\begin{array}{c}0.32 \\
{[0.387]}\end{array}$ & $\begin{array}{c}0.32 \\
{[0.384]}\end{array}$ & $\begin{array}{l}-0.782^{*} \\
{[0.387]}\end{array}$ & $\begin{array}{l}-0.782^{\star} \\
{[0.387]}\end{array}$ \\
\hline Hs Luz 3er. Tr X Musulman (=1) & $\begin{array}{c}0.205 \\
{[0.392]}\end{array}$ & $\begin{array}{c}0.165 \\
{[0.370]}\end{array}$ & $\begin{array}{c}0.389 \\
{[0.614]}\end{array}$ & $\begin{array}{c}0.262 \\
{[0.552]}\end{array}$ & $\begin{array}{c}0.96 \\
{[0.758]}\end{array}$ & $\begin{array}{c}0.96 \\
{[0.758]}\end{array}$ \\
\hline Hs Luz 2er. Tr X Musulman (=1) & $\begin{array}{c}-0.164 \\
{[0.220]}\end{array}$ & $\begin{array}{l}-0.171 \\
{[0.218]}\end{array}$ & $\begin{array}{l}-0.323 \\
{[0.334]}\end{array}$ & $\begin{array}{l}-0.334 \\
{[0.332]}\end{array}$ & $\begin{array}{c}-0.229 \\
{[0.828]}\end{array}$ & $\begin{array}{l}-0.229 \\
{[0.828]}\end{array}$ \\
\hline Hs Luz 1er Tr. X Musulman (=1) & $\begin{array}{l}-0.445 \\
{[0.367]}\end{array}$ & $\begin{array}{l}-0.447 \\
{[0.367]}\end{array}$ & $\begin{array}{l}-0.598 \\
{[0.612]}\end{array}$ & $\begin{array}{l}-0.576 \\
{[0.600]}\end{array}$ & $\begin{array}{c}0.336^{\star * *} \\
{[0.069]}\end{array}$ & $\begin{array}{c}0.336^{\star \star *} \\
{[0.069]}\end{array}$ \\
\hline Hombre & $\begin{array}{l}72.970^{\star \star *} \\
{[24.741]}\end{array}$ & $\begin{array}{l}70.913^{\star * *} \\
{[24.691]}\end{array}$ & $\begin{array}{l}91.966^{* * *} \\
{[29.224]}\end{array}$ & $\begin{array}{l}91.833^{* \star \star *} \\
{[29.249]}\end{array}$ & $\begin{array}{l}113.873^{* *} \\
{[40.654]}\end{array}$ & $\begin{array}{l}113.873^{* *} \\
{[40.654]}\end{array}$ \\
\hline Observaciones & 58,702 & 58,702 & 37,466 & 37,466 & 17,240 & 17,240 \\
\hline R- Cuadrado & 0.009 & 0.013 & 0.016 & 0.027 & 0.022 & 0.022 \\
\hline Numero de Madres & 57,177 & 57,177 & 36,487 & 36,487 & 16,786 & 16,786 \\
\hline Media de Peso al nacer & 3100 & 3100 & 3100 & 3100 & 3000 & 3000 \\
\hline Joint significance Coef & 72.32 & 70.3 & 91.13 & 91.1 & 113.3 & 113.3 \\
\hline Joint significance SE & 24.64 & 24.6 & 29.14 & 29.18 & 40.96 & 40.96 \\
\hline T-test & 2.935 & 2.858 & 3.127 & 3.122 & 2.766 & 2.766 \\
\hline Efecto Fijo por Madre & $\mathrm{Si}$ & $\mathrm{Si}$ & $\mathrm{Si}$ & $\mathrm{Si}$ & $\mathrm{Si}$ & $\mathrm{Si}$ \\
\hline
\end{tabular}

Nota: Los desvios estándar se expresan entre paréntesis. ${ }^{* * *} p<0.01,{ }^{* *} p<0.05,{ }^{*} p<0$. Los desvios fueron clusterados a nivel de País.

\section{Reversibilidad en el peso}

En esta sección se intenta responder a la cuestión sobre si los efectos de los déficits nutricionales durante la gestación, que fueron hallados en el peso al nacer de los niños, persisten más allá de los 24 meses. Existe un consenso en la literatura en general (véase por ejemplo Majid, 2012), en clasificar los efectos del comportamiento analizado en consecuencias de largo plazo (como la reducción de horas de trabajo y la diabetes tipo 2 en la tercera edad) y aquellas que son observables solo en el de corto plazo (como el peso al nacer).

En la sección 6 se presentó evidencia acerca de la perdurabilidad de los efectos de ayunar hasta los 24 meses. Sin embargo, dado que las encuestas DHS cuentan con 
mediciones de los niños de hasta 60 meses, se intentó evaluar el impacto de la déficit nutricional provocado por el cumplimiento del Ramadán, expresándolo en términos de gramos por día, en los niños con edades de 54 a 60 meses (4,5 a 5 años) mediante la regresión del peso de dichos individuos en todas las medidas de Horas de Exposición al Ramadán. No se encontró ningún coeficiente de regresión significativamente distinto de cero, no hallándose evidencia que respalde la hipótesis del efecto del déficit nutricional en este grupo. Por lo tanto, la ausencia de resultados en este panel sugiere que los efectos de ayunar en alguno de los períodos seleccionados de la gestación se diluyeron con el paso del tiempo. Esta conclusión, es consistente con la literatura, y más específicamente con los resultados encontrados por Almond y Mazunder (2011) y Majid (2012) para el peso. ${ }^{10}$ La evidencia de estos trabajos, sin embargo, señaló que existen efectos en el largo plazo, los cuales se evidencian por discapacidades físicas, déficits en la salud y menor calidad de vida, entre otros aspectos.

\section{Conclusiones}

Este trabajo empleó un conjunto de variables que miden las Horas de exposición al mes de ayuno islámico del Ramadán con el fin de realizar un análisis detallado del impacto de un déficit nutricional sobre el peso al nacer (y hasta los 24 meses). Para ello se utilizó una base de datos de panel con todas las muestras y las observaciones disponibles en las encuestas DHS. Dado que ayunar en este período constituye una actividad sólo realizada por aquellos individuos que profesan el Islam, la configuración final de estudio es un experimento natural que busca evaluar si existe una relación de causalidad entre las dos: el peso al nacer y la exposición intrauterina. En el mes de estudio los dos canales de transmisión son el ayuno diurno y el desorden tanto social como alimenticio provocado por una sociedad que se revoluciona a partir del evento religioso.

Al igual que toda la literatura empírica previa acerca de déficits nutricionales y en línea con la teoría médica, este trabajo encontró que el efecto promedio de ayunar el período completo es de 300 gr., lo que representa un 9\% la media muestral. Las estimaciones fueron realizadas con las variables de Horas de Exposición uterina al Ramadán tanto a

\footnotetext{
${ }^{10}$ En contraposición, los niños afectados por la confluencia entre el Ramadán y la gestación muestran efectos en altura, efectos que son observables aún en la etapa de adultos (Karimi, 2013)
} 
nivel trimestral como mensual. En todas, los resultados son consistentes con efectos en el segundo y tercer trimestre de gestación puesto que los coeficientes son negativos y significativos.

Si bien existe poca información disponible acerca de los costos en África, Sicuri (2011) calculó el monto en el que incurren las familias rurales de Mozambique para que el niño aumente 100 gr en US\$36,90 ${ }^{11}$ para el 2012. Por lo tanto, estos individuos deben gastar aproximadamente US\$60 en cuidados para la salud de los niños musulmanes. Este monto representa un $10 \%$ de su salario anual según datos de la ONU. ${ }^{12}$

Muchos autores, como Eiwijk (2011), Majid (2012) o Almond y Mazunder (2011), buscaron medir los efectos a más largo plazo. El presente trabajo trató de aproximarse al mediano plazo utilizando las variables de peso y de Z-scores de la OMS 2006 hasta los 24 meses de vida del niño. Cuando se considera al grupo de países con más de $10 \%$ de población musulmana, se observó que el efecto más importante es de ayunar en el primer trimestre de la gestación. Los restantes no mostraron niveles elevados de significatividad.

Estos resultados ponen de relieve la necesidad de mantener un alto grado de cuidado de la salud de las mujeres embarazadas puesto que los efectos en el peso se perciben incluso dos años después del nacimiento. En el mundo musulmán, podrían contribuir a revisar la política sobre el permiso para no ayunar a las madres. Sin embargo, debe considerarse también que el mecanismo de accelerated starvation no solo ocurre con el Ramadán. Cuando una mujer no se alimenta de forma apropiada y saltea una comida, se genera el mismo proceso. De esto se desprende que es de suma importancia informar a las mujeres sobre posibles consecuencias de no llevar una vida ordenada durante este período.

Por último, esta tesis ofrece un amplio espectro para el análisis futuro. En primer lugar, la poca información sobre costos disponible para los países africanos hace que sea muy difícil estimar el impacto económico y en los niveles de utilidad de las familias afectadas por el nacimiento de un niño con bajo peso al nacer. En lo que respecta al peso, podría resultar interesante evaluar los efectos de trasmisión entre madres e hijos que sufrieron exposición al déficit nutricional mientras se gestaban. Asimismo, las

\footnotetext{
${ }^{11} \mathrm{E}$ l desglose de este costo comprende US\$24,12 más n incremental de 53\% por cada 100 gr de aumento.

12 Este dato fue encontrada en este sitio web el 22 de Noviembre de 2014: https://data.un.org/CountryProfile.aspx?crName=Mozambique
} 
encuestas DHS son actualizadas anualmente brindando más datos y más países para analizar efectos en el peso o similares en futuras investigaciones.

\section{Bibliografía}

Almond, Douglas, Kenneth Y. Chay and David S. Lee. "The Costs Of Low Birth Weight," Quarterly Journal of Economics, 2005, v120(3,Aug), 1031-1083.

Almond, D., Mazumder, B. 2005,The 1918 influenza pandemic and subsequent health outcomes: an analysis of SIPP data, American Economic Review, 95(2), 258-62.

Almond, D y Mazunder, B. 2011. Health Capital and the Prenatal Enviroment: The effect of Ramadan Observance during Pregnancy. American Economic Journal: Applied Economics. 3, $56-80$

Almond, Douglas, Bhashkar Mazumder, and R. van Ewijk, 2011, Fasting during pregnancy and children's academic performance,"NBER Working Paper, No. 17713.

Camacho, A. 2008. Stress and Birth Weight: Evidence from Terrorist Attacks. Amer. Econ. Review: Papers \& Proceedings. 98, 511-515.

Cruces, G; Glüzmann, P; Lopez Calva L F. 2011. Economic Crises, Maternal and Infant Mortality, Low Birth Weight and Enrollment Rates: Evidence from Argentina's Downturns. CEDLAS-FCE-UNLP. Documento de Trabajo Nro. 121

Ewik, R. 2011. Long-Term Health Effect on the nect generation of Ramadan fasting during pregnancy. Journal of Health Economics. 30, 1246-1260

Hodek1, J; Schulenburg, M and Mittendor, T 2011 Measuring economic consequences of preterm birth - Methodological recommendations for the evaluation of personal burden on children and their caregivers. Health Economics Review

Joosoph, J; Abu, J; Yu S. L. 2004. A Survey of fasting during pregnancy. Singapore Med. J.45, 583-586.

Karimi., S. 2014. The Effect of Malnutrition In Utero on Height: Ramadan as a Natural Experiment, Job Market Paper Universitu of Illinois at Urbana-Champign

Majid, M. F., 2012,The persistent efects of in utero nutrition shocks over the life cycle: evidence from Ramadan fasting in Indonesia,"Job Market Paper, University of California at Riverside.

Sicuri E, Bardají A, Sigauque B, Maixenchs M, Nhacolo A, et al. (2011) Costs Associated with Low Birth Weight in a Rural Area of Southern Mozambique. PLoS ONE 6(12): e28744. doi:10.1371/journal.pone.0028744

Tongo O., Orimadegun A., Ajayi S. y Akinyinka O. 2008 The Economic Burden of Preterm/Very Low Birth Weight Care in Nigeria. Oxford University Press 
Fernández Long, M. E. y Hurtado, R. 2014. "Planilla de cálculo de variables astronómicas VarAst1.0." Centro de Información Agroclimática (CIAg) de la Facultad de Agronomía Universidad de Buenos Aires. (http://www.agro.uba.ar/centros/ciag) 